\title{
Does the trait anxiety affect the dental fear?
}

\section{Yusuf Cetin DOGANER ${ }^{(a)}$ \\ Umit AYDOGAN(b) \\ Hande Ucler YESIL ${ }^{(c)}$ \\ James Edwin ROHRER(d) \\ Mark Douglas WILLIAMS(e) \\ David Charles AGERTER ${ }^{(f)}$}

(a) National Defense University, Turkish Military Academy, Primary Care Examination Center, Ankara, Turkey.

(b) University of Health Sciences, Gulhane Medical Faculty, Department of Family Medicine, Ankara, Turkey.

(c)Diskapi Yildirim Beyazit Training and Research Hospital, Oral Health and Dental Treatment Center, Ankara, Turkey.

(d)Walden University, School of Health Sciences, Minneapolis, MN, USA.

(e) Mayo Clinic, Department of Psychiatry and Psychology, Rochester, MN, USA.

(f) Mayo Clinic, Department of Family Medicine, Rochester, MN, USA.

Declaration of Interests: The authors certify that they have no commercial or associative interest that represents a conflict of interest in connection with the manuscript.

Corresponding Author:

Yusuf C. Doganer, MD

E-mail: ycetindoganer@hotmail.com

https://doi.org/10.1590/1807-3107BOR-2017.vol31.0036

Submitted: Jun 16, 2015

Accepted for publication: Mar 27, 2017

Last revision: Apr 05, 2017
Abstract: The aims of the present study were to evaluate possible associations between trait anxiety, dental fear and the predictors of these interactions including demographic characteristics and dental history of patients applied to the dental care center in Ankara, Turkey. A sample of 607 participants (mean age: $21.02 \pm 2.32$ ) responded to a Turkish version of the Modified Dental Fear Survey (MDFS), the State-Trait Anxiety Inventory (STAI-T) and a questionnaire regarding previous negative dental experience. Multiple logistic regression analysis was used to identify the association between dental fear and the independent variables including trait anxiety, age groups, education level, dental visit frequency, experience and the source of dental knowledge. There was a trend for increasing in trait anxiety scores with greater levels of dental fear in a medium level of the dental fear group (OR = 1.055, 95\%CI [1.025-1.086]; $\mathrm{p}<0.001)$ and in a high level of the dental fear group (OR $=1.090$ [1.057-1.124]; $p<0.001)$. Comparing to the low level of dental fear group; participants of medium dental fear level intended more likely to go to the dentist when they have a complaint instead of regularly going (odds ratio; OR = 3.177, 95\%CI [1.304-7.741]; $\mathrm{p}=0.011$ ). Participants of high dental fear level tended to be less likely to have experienced no problem (OR $=0.476,95 \% \mathrm{CI}[0.284-0.795] ; \mathrm{p}=0.005)$ than the low level of the dental fear group. We strongly indicate that higher dental fear scores have a predisposition of having high trait anxiety scores. Unpleasant dental experiences increased the risk for high dental fear levels. Patients with dental fear tended only to visit a dentist when necessary, avoiding regular visits.

Keywords: Dental Health Services; Behavioral Sciences; Public Health Dentistry.

\section{Introduction}

Anxiety disorders include measures both of state and trait anxiety. The condition of state anxiety is just a transitional mood varying from mild to severe level of intensity that is meant to describe consciously perceived feelings and an autonomic response. However, the condition of trait anxiety link with a personality trait and are stable during a person's lifetime. ${ }^{1}$ State anxiety is relevant to associate with patient's present stage of anxiety; whilst trait anxiety is relevant to reveal the patient's lifelong anxiety level. ${ }^{2}$

Dental fear is highly prevalent and affects both personal health and public health. In various population studies, the prevalence of high dental 
fear differs from 10 to $20 \%{ }^{3,4}$ People having high dental fear hold more untreated caries and extensive dental treatment need. ${ }^{5}$ These patients also have the poorer oral health-related quality of life comparing to others having less dental fear. ${ }^{6,7}$

Dental fear could be described as a state anxiety condition due to the obvious challenges involved in dental treatment procedures and shared knowledge or rumors of traumatic dental histories. Some relevant variables include any traumatic experiences in the childhood, negative attitudes about dental care in the family, and negative perceptions and expectations after the painful dental treatment. ${ }^{8}$ Individuals with a high level of dental fear may intend to delay their dental appointments, perpetuating a vicious cycle of dental fear. Contents of this cycle include avoidance, increased the degree of dental problems, and may lead to feelings of embarrassment and inferiority.,10,11 Beyond the dental health state of the individual, dental fear also could affect the dentist-patient relation causing to misdiagnosis and redundant delay during the treatment process. ${ }^{12}$

The interaction between dental fear and trait anxiety has been examined in different types of studies. ${ }^{13,14}$ However, to our knowledge, the relationship between trait anxiety and dental fear has not been investigated using the instrument of the Modified Dental Fear Survey. The current study sought to determine whether trait anxiety impacts the dental fear status of the young individuals applied to oral and dental care unit of primary care. In addition, possible different confounders which might impact the presence or absence of dental fear were examined. It was hypothesized that: a) dental fear would be closely and significantly associated with an individual's trait anxiety level; b) the stage of dental fear would be affected by some potential independent variables including age groups, educational level, past negative dental experience, dental visit frequencies, sources of dental knowledge.

\section{Methodology}

\section{Population and study design}

The present cross-sectional study was carried out between April and September 2012 at the Oral Health and Diagnosis unit of the primary care examination center that serves especially young individuals aged between 14-30 years in Ankara, Turkey. Some of the patients waiting for dental care in the lounge refused to participate due to not having sufficient time or not agreeing to fill out the questionnaires. Hence, 607 participants were enrolled in the study. Participants getting treatment for any psychiatric disorders in their medical histories were excluded from the study. All recruited patients gave informed consent after hearing about the aims and procedures involved in the study. Ethical approval was taken from the institutional ethics board.

\section{Questionnaires}

Participants responded to the State -Trait Anxiety Inventory (STAI-T) ${ }^{15}$, the Turkish version of Modified Dental Fear Survey (MDFS) ${ }^{16}$ and a questionnaire regarding previous negative dental experience. All measurement tools and questionnaire were self-administered.

Age groups were scored in three categories $(<20$ years $/ 20-25$ years/ $>25$ years). Educational levels were scored as $<12$ years or $>12$ years and gender was scored as male or female. The questionnaire including information about frequencies of previous dental visits (Never / When has a complaint / regularly - every six months), state of having any problem (Yes / No), the source of dental knowledge from (parents / dentist / TV-internet) and each person was asked demographic characteristics.

Trait anxiety was measured by the trait form of the Turkish version of the State-Trait Anxiety Inventory (STAI-T). Trait anxiety tends to predict to what extent the patient is meticulous in his/her habits and to what extent that a person exhibits an anxious state even in the absence of an external stressor. The Turkish version of the STAI-T consists of 20 questions, 7 items indicating trait anxiety absent and 13 indicating trait anxiety present. Each item is rated on a 4-point scale: 1 (almost never), 2 (sometimes), 3 (often) and 4 (almost always). Anxiety-absent items are scored in reverse during the calculation of individuals' total scores. The total score of the scale ranges from 20 to 80 . Higher scores reflect higher levels of trait anxiety and lower scores reflect lower levels of trait anxiety. This inventory has been widely used for the evaluation of trait anxiety in numerous clinical contents. ${ }^{15}$ 
The Modified Dental Fear Survey (MDFS) was originally converted from the Dental Hygiene Fear Survey (DHFS). Since the lack of dental hygienists professionals in Turkish dental health system, dental hygiene processes are carried out by dentists. Though this slight alteration in dental interventions, dental hygiene treatment may also elicit various dental fear. DHFS was initially improved as a hypothetical model of dental fear by investigators of the University of Washington (UW) Dental Fears Clinic. ${ }^{17}$ Later in another study, the DHFS was validated as a reliable tool to investigate dental fear and the relationship between the dental fear and variables including age, gender, and avoidance of dental care..$^{18}$ The survey was designed using a Likert rating scale ranging from $1=$ "not at all" to $5=$ "very much" for each item. It is composed of 16 questions to determine the dental fear and higher total scores depict the greater level of dental fear. The term of "dental hygienist" in the original version was converted to "dentist" in line with professions current in Turkish dental health care system. Additionally, $11^{\text {th }}$ item in the survey was excluded from the survey during the testing of reliability and validity of the Turkish version. ${ }^{16}$

\section{Statistical analysis}

Statistical analyses were conducted by using the software Statistical Package for the Social Sciences (SPSS for Windows, version 22.0, SPSS Inc., Chicago, IL, USA) program. Data analysis included descriptive statistics, and the outcome was tested by bivariate and multivariate analysis. Mann-Whitney $U$ test and Kruskal-Wallis test were implemented to compare the groups due to unequal variances. Differences with $p<0.05$ were considered as statistically significant.

Multiple logistic regression analysis was conducted using trichotomized dental fear as the dependent variable (low level of dental fear $=0$, medium level of fear $=1$, and high level of fear = 2). The following independent variables were used in the logistic regression model and were dichotomized to 0 and 1 as follow: education level $(>12$ years $=1)$, ever experienced a problem (Yes $=1)$; were trichotomized into 0 to 2 as follow: age groups $(>25$ years $=2,20-25$ years $=1$, $<20$ years $=0$ ), dental visit frequency (regularly $=2$, has a complaint $=1$, never $=0$ ), sources of dental knowledge $(\mathrm{TV}$-internet $=2$, dentist $=1$, parents $=0$ )

\section{Results}

Ninety-three patients refused to participate in the study (Response rate $=86.7 \%$ ). The final sample size was composed of 607 volunteers, aged $21.02 \pm 2.32$ (14-30 years), and $96.9 \%$ were men. Of the participants, $74.8 \%(n=454)$ were in the $20-25$ year-aged group, $56.7 \%(n=344)$ were educated more than 12 years. Table 1 depicts the demographic characteristics of the participant patients.

In the total sample, $76.9 \%(n=467)$ had been to dental visit where they had a complaint about their oral and dental health. Of those people applying for dental care and treatment, $28.8 \%(n=145)$ had a problem in their past negative dental experiences. It is revealed that $48.4 \%(n=294)$ of the patients got their knowledge about dental health and care from their own dentists (Table 1).

High scores in the MDFS survey depict the high level of dental fear. According to the distribution of total scores, the MDFS survey's results were stratified into 3 equal levels of dental fear such as low (14-18), medium (19-27), high (28-70). The distribution of dental fear in the participant group that regularly visit their dentists was 9.3\% $(n=18)$ low level, 3.8\% $(\mathrm{n}=8)$ medium level and $4.9 \%(\mathrm{n}=10)$, while the

Table 1. Descriptive features of the participants $(n=607)$.

\begin{tabular}{lcc}
\hline Variable & $\mathrm{n}$ & $\%$ \\
\hline Age groups & & \\
$\quad<20$ years & 121 & 19.9 \\
$20-25$ years & 454 & 74.8 \\
$\quad>25$ years & 32 & 5.3 \\
Gender (male) & 588 & 96.9 \\
Education levels & & \\
$\quad<12$ years & 263 & 43.3 \\
$\quad>12$ years & 344 & 56.7 \\
Dental visit frequency & & \\
$\quad$ Never & 104 & 17.1 \\
$\quad$ Having a complaint & 467 & 76.9 \\
$\quad$ Regularly & 36 & 5.9 \\
Experienced a problem? ${ }^{*}$ & & \\
$\quad$ No & 358 & 71.2 \\
$\quad$ Yes & 145 & 28.8 \\
The source of dental knowledge & & \\
$\quad$ Parents & 138 & 22.7 \\
Dentist & 294 & 48.4 \\
TV-internet & 175 & 28.8 \\
\hline
\end{tabular}

${ }^{*} n=503$ (patients who never have a dental visit were excluded). 
$83.8 \%(n=176)$ of the participants who go to the dental health and care center just when they have a relevant complaint tend to show a medium level of dental fear $(p=0.015)$. Of the participants who have experienced a problem in their dental history, the distribution of dental fear severity as follows: $23.4 \%(n=36)$ low, $22.3 \%$ $(\mathrm{n}=41)$ medium, 41.2\% $(\mathrm{n}=68)$ high $(\mathrm{p}<0.001)$ (Table 2$)$. The analysis revealed that there were statistically significant differences between the level of dental fear and participants' dental visit frequencies and having an unpleasant experience. Statistically significant differences could not be found between the levels of dental fear in terms of age groups, genders, educational levels, sources of knowledge ( $p>0.05)$.

The mean/median score in the MDFS was higher in the group that experienced a dental problem comparing to did not $(28.45 / 26 \pm 11.61$ vs. $24.22 / 21 \pm 9.87)(\mathrm{p}<0.001)$. While the participants who had learned about dental health from TV-internet showed the highest mean/median score in the MDFS (27.21/24 \pm 11.72$)$, the mean/median of total scores of participants who had learned about dental health from dentists and parents were lower $(25.65 / 22 \pm 11.10,23.34 / 21.5 \pm 8.21)(p=0.040)($ Table 3$)$.

Table 2. Relationship between dental history and categorized total score of the MDFS ( $\mathrm{n}=607)$.

\begin{tabular}{|c|c|c|c|c|}
\hline \multirow{2}{*}{ Variable } & \multicolumn{3}{|c|}{ Categorized total score of the MDFS [n (\%)] } & \multirow{2}{*}{$p^{*}$} \\
\hline & Low & Medium & High & \\
\hline \multicolumn{5}{|l|}{ Dental visit frequency } \\
\hline Never & $39(20.2)$ & $26(12.4)$ & $39(19.1)$ & \\
\hline Having a complaint & $136(70.5)$ & $176(83.8)$ & $155(76.0)$ & 0.015 \\
\hline Regularly & $18(9.3)$ & $8(3.8)$ & $10(4.9)$ & \\
\hline \multicolumn{5}{|c|}{ Experienced a problem? ${ }^{* *}$} \\
\hline No & $118(76.6)$ & $143(77.7)$ & $97(58.8)$ & $<0.001$ \\
\hline Yes & $36(23.4)$ & $41(22.3)$ & $68(41.2)$ & \\
\hline
\end{tabular}

${ }^{*}$ Chi-Square test; ${ }^{* *} \mathrm{n}=503$ (patients who never have a dental visit were excluded).

Table 3. Dental fear and trait anxiety comparison in terms of independent variables $(n=607)$.

\begin{tabular}{|c|c|c|c|c|}
\hline \multirow{2}{*}{ Variable } & MDFS total score & \multirow{2}{*}{$\mathrm{P}^{*}$} & STAI-T Anxiety & \multirow{2}{*}{$\mathrm{P}^{*}$} \\
\hline & mean/median (sd) & & mean/median (sd) & \\
\hline \multicolumn{5}{|l|}{ Age groups } \\
\hline$<20$ years & $25.02 / 22(9.52)$ & & $40.37 / 40(7.57)$ & \\
\hline $20-25$ years & $25.56 / 22(10.94)$ & 0.547 & $42.35 / 43(8.55)$ & 0.017 \\
\hline$>25$ years & $27.81 / 25$ (12.80) & & $43.91 / 44.5(6.51)$ & \\
\hline \multicolumn{5}{|l|}{ Education levels } \\
\hline$<12$ years & $26.61 / 23(11.24)$ & \multirow{2}{*}{0.056} & $44.36 / 46(8.46)$ & \multirow{2}{*}{$<0.001$} \\
\hline$>12$ years & $24.78 / 22(10.36)$ & & $40.27 / 40(7.74)$ & \\
\hline \multicolumn{5}{|l|}{ Dental visit frequency } \\
\hline Never & $26.23 / 22.5(11.78)$ & & $44.73 / 46(8.05)$ & \\
\hline Having a complaint & $25.64 / 22(10.50)$ & 0.080 & $41.43 / 41(8.18)$ & 0.001 \\
\hline Regularly & $22.75 / 18.5(11.22)$ & & $42.22 / 42.5(9.25)$ & \\
\hline \multicolumn{5}{|c|}{ Experienced a problem? ${ }^{* *}$} \\
\hline No & $24.22 / 21(9.87)$ & $<0.001$ & 40.94/41 (8.18) & 0.032 \\
\hline Yes & $28.45 / 26(11.61)$ & & $42.83 / 42(8.31)$ & \\
\hline \multicolumn{5}{|c|}{ The source of dental knowledge } \\
\hline Parents & $23.34 / 21.5(8.21)$ & & $41.33 / 41(8.77)$ & \\
\hline Dentist & 25.65/22 (1 1.10) & 0.040 & $42.15 / 42(8.28)$ & 0.470 \\
\hline TV-internet & $27.21 / 24(11.72)$ & & $42.42 / 42(7.97)$ & \\
\hline
\end{tabular}

${ }^{*}$ Dichotomized groups were analyzed with Mann-Whitney $\mathrm{U}$ test, trichotomized groups were analyzed with Kruskal-Wallis test; ${ }^{* *} \mathrm{n}=503$ (patients who never have a dental visit were excluded); \# Gender differences were not shown regarding majority of participants (96.9\%) were composed by males; MDFS: modified dental fear survey; STAI-T: state-trait anxiety inventory (Trait) 
Other independent variables including age groups, education levels, dental visit frequency could not be detected as statistically significant $(\mathrm{p}>0.05)$.

The statistical significances were ascertained in the assessment of mean/median scores of STAI-T survey in the group comparison of age, educational levels, dental visit frequency, a problem experienced $(p=0.017, p<0.001, p=0.001, p=0.032)$. A significant difference was not found regarding the source of dental knowledge $(p>0.05)$ (Table 3).

Multivariate logistic regression analysis showed that the trait anxiety and the dental visit frequency in a medium level of the dental fear group, and the trait anxiety and the status of experienced a dental problem were significantly different comparing to low level of dental fear. Comparing to the low level of dental fear, participants of medium dental fear level intended more likely to go to the dentist when they have a complaint instead of regularly going (odds ratio; OR = 3.177, 95\%CI [1.304-7.741]; $\mathrm{p}=0.011$ ). Comparing to the low level of dental fear, participants of high dental fear level tended to be less likely to have experienced no problem $(\mathrm{OR}=0.476$, $95 \%$ CI [0.284-0.795]; $\mathrm{p}=0.005)$. There was a trend for increasing in STAI-T scores with greater levels of dental fear in a medium level of the dental fear group $(\mathrm{OR}=1.055,95 \% \mathrm{CI}[1.025-1.086] ; \mathrm{p}<0.001)$ and in a high level of the dental fear group $(\mathrm{OR}=1.090$ [1.057-1.124]; $\mathrm{p}<0.001$ ) (Table 4).

\section{Discussion}

Although we initially detected statistical differences regarding the source of dental knowledge in MDFS scores, and statistical differences by age groups and education levels in the trait anxiety; these differences disappeared after regression analysis. The remaining essential finding of the present study was a substantial association between the trait anxiety and levels of dental fear. It was reported that the ascending dental fear related to higher trait anxiety levels. Moreover, the relationship with dental visit frequency and any experienced dental problem regarding levels of dental fear was ascertained as statistically significant. In addition, to our knowledge, the present study is the first study which is conducted for examining the
Table 4. Multivariate logistic regression analysis of the MDFS [Medium (36.6\%, $\mathrm{n}=184$ ) or High dental fears (32.8\%, $\mathrm{n}=165)$ vs Low dental fear $(30.6 \%, \mathrm{n}=154)]$.

\begin{tabular}{|c|c|c|}
\hline Trait anxiety & OR $(95 \% \mathrm{Cl})$ & $p$ \\
\hline Medium & $1.055(1.025-1.086)$ & $<0.001$ \\
\hline \multicolumn{3}{|l|}{ Age groups } \\
\hline$<20$ years & $1.100(0.376-3.217)$ & 0.862 \\
\hline $20-25$ years & $1.039(0.386-2.796)$ & 0.939 \\
\hline$>25$ years & Reference & - \\
\hline \multicolumn{3}{|l|}{ Education } \\
\hline$<12$ years & $0.810(0.506-1.298)$ & 0.381 \\
\hline$>12$ years & Reference & - \\
\hline \multicolumn{3}{|l|}{ Dental visit frequency } \\
\hline Having a complaint & $3.177(1.304-7.741)$ & 0.011 \\
\hline Regularly & Reference & - \\
\hline \multicolumn{3}{|l|}{ Experienced a problem? } \\
\hline No & $1.110(0.655-1.881)$ & 0.698 \\
\hline Yes & Reference & - \\
\hline \multicolumn{3}{|c|}{ The source of dental knowledge } \\
\hline Parents & $1.114(0.617-2.010)$ & 0.720 \\
\hline Dentists & $1.191(0.694-2.044)$ & 0.526 \\
\hline TV-internet & Reference & - \\
\hline High & $1.090(1.057-1.124)$ & $<0.001$ \\
\hline \multicolumn{3}{|l|}{ Age groups } \\
\hline$<20$ years & $1.184(0.395-3.550)$ & 0.763 \\
\hline $20-25$ years & $0.970(0.355-2.647)$ & 0.952 \\
\hline$>25$ years & Reference & - \\
\hline \multicolumn{3}{|l|}{ Education } \\
\hline$<12$ years & $0.990(0.605-1.620)$ & 0.968 \\
\hline$>12$ years & Reference & - \\
\hline \multicolumn{3}{|l|}{ Dental visit frequency } \\
\hline Having a complaint & $2.035(0.855-4.843)$ & 0.108 \\
\hline Regularly & Reference & - \\
\hline \multicolumn{3}{|l|}{ Experienced a problem? } \\
\hline No & $0.476(0.284-0.795)$ & 0.005 \\
\hline Yes & Reference & - \\
\hline \multicolumn{3}{|c|}{ The source of dental knowledge } \\
\hline Parents & $0.539(0.286-1.014)$ & 0.055 \\
\hline Dentists & $0.839(0.484-1.455)$ & 0.533 \\
\hline TV-internet & Reference & - \\
\hline
\end{tabular}

${ }^{*} n=503$ (patients who never have a dental visit were excluded in multivariate logistic regression analysis).

association between dental fear and trait anxiety in the dental health center of primary care and by using the MDFS survey.

Since the absence of studies with which to compare our study regarding the relationship between dental fear 
and trait anxiety, we preferred to compare the present study with previous ones in terms of the association of dental anxiety and trait anxiety. Previous studies have been inconsistent on this subject. Fuentes et al. ${ }^{19}$ emphasized that dental anxiety is a particular condition with its own features independent of trait anxiety. They detected a statistically significant relationship between high dental anxiety scores and high trait anxiety scores, whilst not finding an association between high trait anxiety scores and high dental anxiety scores. Okawa et al. ${ }^{20}$ found no difference in the level of preoperative anxiety between the high- and low-trait anxiety groups. In contrast, statistical significant differences between dental anxiety and trait anxiety have also been detected. A statistical significant association was indicated between trait and dental anxiety in a study conducted among patients who were getting treated specifically for dental anxiety. ${ }^{13}$ Hägglin et al. ${ }^{14}$ found similar results even though using a different instrument for evaluating the dental anxiety. A study conducted by Lago-Méndez et al. ${ }^{21}$ suggested that trait anxiety may be an efficient psychometric parameter for patients' predisposition to dental anxiety. Similar to the previous studies that were found a positive correlation between the dental and trait anxiety, the statistically significant difference was detected between dental fear levels and trait anxiety scores in the present study.

Age also had an effect on dental and trait anxiety. Armfield et al. depicted that a patient's age correlated with anxiety and of the participants, adults aged between 40-64 years had the highest dental anxiety stage. ${ }^{4}$ Age was one of the most significant modifiers in Pohjola et al. ${ }^{3}$ study indicating this interaction of both measurement scores. Fabián et al. ${ }^{22}$ similarly concluded their studies finding an association between dental and trait anxiety states. Locker et al. ${ }^{23}$ emphasized that young individuals have a high level of dental anxiety had higher rates of psychological disorders in their study. Our results were particularly supporting the previous studies. We assessed a significant relationship between the STAI-T scores and age groups, however, could not find a difference in terms of dental fear levels.

In most of the studies, the close relationship between negative dental experience in childhood and dental anxiety level wasfound out. Such experiences have been found closely associated with an increased sense of pain and negative beliefs about dental treatment. ${ }^{24}$ Levin et al. ${ }^{25}$ found significant differences in the prevalence of high dental anxiety between participants treated by regular dental visits in childhood and those treated sporadically. Oliveira et al. ${ }^{11}$ revealed that negative dental experiences in childhood were related to dental avoidance of the patients. Similarly, having a past perceived bad experience was ascertained highly associated with the level of dental anxiety in another study. ${ }^{26}$ Heaton et al. ${ }^{27}$ also reported on the fact that higher dental fear survey scores were related to past avoidance of dental care regarding a bad experience for the prediction of higher observed anxiety. The results of the present study were in agreement with previous studies indicating the negative dental experience in their lives had significantly associated higher scores of dental fear. Since most individuals have onset and develop their dental fears during the childhood period, more attention should be paid by the dentists and parents at this stage. In severe cases, dental trauma cannot be only the cause of the poor oral health, but even also impact mental health by the genesis of posttraumatic stress disorders. ${ }^{28}$

The regularity of dental visits was thought another determinant in the development of dental fear. Dental anxiety was reported to be higher in irregular dental visitors comparing to regular subjects. ${ }^{29}$ Similar percentages like in our studies, Taani ${ }^{8}$ found in his previous study that the majority of his study sample consisting of the young adult population was reported applying to the dentist only in emergency cases. In another assessment of dental visit frequency, irregular attenders were found to be more likely to be afraid of visiting a dentist, especially among 65-year-olds. ${ }^{3}$ In another study evaluating the strongest predictor of the onset of dental anxiety, the avoidance of dental care was found the substantial factor. ${ }^{30}$ Contrary to these studies, Armfield et al. ${ }^{31}$ defined that there were no significant differences in dental fears between non-avoidant patients and avoidant individuals. Our findings in the present study indicated that the participants who never visited a 
dentist, thus behaving in an avoidant manner have higher trait anxiety scores than non-avoiders. This included regular visitors and the participants who only attended a visit when they have a specific dental complaint. The existence of being the difference in trait anxiety scores whilst not in the MDFS scores could identify the power of general feature of personality over the individuals' genesis of different fears.

\section{Study limitation}

A study limitation was that all participants reported self-administered surveys. Various biases may occur regarding the augmenting traumatic experiences. Hence, the results should be evaluated with caution. Secondly, almost most of the participants were male, because of the reason that the current study was carried out in the military health-care unit. Finally, our study was conducted in a single oral and dental health center of the primary care. Therefore, findings may not be generalizable to other dental care centers in Turkey. Multicenter and more detailed studies involving different age groups are required to elucidate the correlation between dental fear and trait anxiety.

\section{References}

1. Akarslan ZZ, Erten H, Uzun O, Işeri E, Topuz O. Relationship between trait anxiety, dental anxiety and DMFT indexes of Turkish patients attending a dental school clinic. East Mediterr Health J. 2010;16(5):558-62.

2. Sari Z, Uysal T, Karaman AI, Sargin N, Ure O. Does orthodontic treatment affect patients' and parents' anxiety levels? Eur J Orthod. 2005;27(2):155-9. https://doi. org/10.1093/ejo/cjh072

3. Pohjola V, Lahti S, Tolvanen M, Hausen H. Dental fear and oral health habits among adults in Finland. Acta Odontol Scand. 2008;66(3):148-53. https://doi. org/10.1080/00016350802089459

4. Armfield JM, Spencer AJ, Stewart JF. Dental fear in Australia: who's afraid of the dentist? Aust Dent J. 2006;51(1):78-85. https://doi.org/10.1111/j.1834-7819.2006.tb00405.x

5. Armfield JM, Slade GD, Spencer AJ. Dental fear and adult oral health in Australia. Community Dent Oral Epidemiol. 2009;37(3):220-30. https://doi.org/10.1111/j.1600-0528.2009.00468.x

6. Crofts-Barnes NP, Brough E, Wilson KE, Beddis AJ, Girdler NM. Anxiety and quality of life in phobic

\section{Conclusion}

Depending on the current study, we strongly emphasize that higher dental fear scores have a predisposition of having high trait anxiety scores even though both conditions are interpreted separately in general. Patients who have higher trait anxiety also are at increased risk for both medium and high dental fear, even after adjusting for other variables. Unpleasant dental experiences increased the risk for high dental fear but not for medium dental fear. Patients with dental fear tended only to visit a dentist when necessary, avoiding regular visits.

Dental practitioners should be informed that patients with trait anxiety are likely to be more fearful of dental services and likely to avoid preventive visits. Patient education materials address the importance of preventive visits should be provided to anxious patients. In addition, after an anxious patient has an unpleasant experience, special outreach efforts may be indicated to encourage preventive services.

\section{Acknowledgements}

None declared.

dental patients. J Dent Res. 2010;89(3):302-6. https://doi. org/10.1177/0022034509360189

7. Gisler V, Bassetti R, Mericske-Stern R, Bayer S, Enkling $\mathrm{N}$. A cross-sectional analysis of the prevalence of dental anxiety and its relation to the oral health-related quality of life in patients with dental treatment needs at a university clinic in Switzerland. Gerodontology. 2012;29(2):e290-6. https://doi.org/10.1111/j.17412358.2011.00465.x

8. Taani DSMQ. Dental fear among a young adult Saudian population. Int Dent J. 2001;51(2):62-6. https://doi. org/10.1002/j.1875-595X.2001.tb00823.x

9. Armfield JM, Stewart JF, Spencer AJ. The vicious cycle of dental fear: exploring the interplay between oral health, service utilization and dental fear. BMC Oral Health. 2007;7(1):1. https://doi.org/10.1186/1472-6831-7-1

10. Moore R, Brødsgaard I, Rosenberg N. The contribution of embarrassment to phobic dental anxiety: a qualitative research study. BMC Psychiatry. 2004;4(1):10. https://doi. org/10.1186/1471-244X-4-10 
11. Oliveira MA, Bendo CB, Ferreira MC, Paiva SM, Vale MP, Serra-Negra JM. Association between childhood dental experiences and dental fear among dental, psychology and mathematics undergraduates in Brazil. Int J Environ Res Public Health. 2012;9(12):4676-87. https://doi.org/10.3390/ ijerph9124676

12. Eli I. Dental anxiety: a cause for possible misdiagnosis of tooth vitality. Int Endod J. 1993;26(4):251-3. https://doi. org/10.1111/j.1365-2591.1993.tb00567.x

13. Hakeberg M, Hägglin C, Berggren U, Carlsson SG. Structural relationships of dental anxiety, mood, and general anxiety. Acta Odontol Scand. 2001;59(2):99-103. https://doi.org/10.1080/000163501750157252

14. Hägglin C, Hakeberg M, Hällström T, Berggren U, Larsson L, Waern $\mathrm{M}$ et al. Dental anxiety in relation to mental health and personality factors. A longitudinal study of middle-aged and elderly women. Eur J Oral Sci. 2001;109(1):27-33. https://doi.org/10.1034/j.16000722.2001.00946.x

15. Oner N, LeCompte A. State-trait anxiety inventory handbook. 2nd ed. Istanbul: Bogazici University Issues; 1985.

16. Ay ZY, Gündogar D, Büyüköztürk S, Gadbury-Amyot C. Dental hygiene fear survey: reliability and validity of the Turkish version. J Dent Educ. 2008;72(2):183-94.

17. Weinstein P, Milgrom P, Getz T. Treating fearful dental patients: a practical behavioral approach. J Dent Pract Adm. J Dent Pract Adm. 1987;4(4):140-7.

18. Gadbury-Amyot CC, Overman PR, Carter-Hanson C, Mayberry W. An investigation of dental hygiene treatment fear. J Dent Hyg. 1996;70(3):115-21.

19. Fuentes D, Gorenstein C, Hu LW. Dental anxiety and trait anxiety: an investigation of their relationship. Br Dent J. 2009;206(8):E17. https://doi.org/10.1038/sj.bdj.2009.253

20. Okawa K, Ichinohe T, Kaneko Y. Anxiety may enhance pain during dental treatment. Bull Tokyo Dent Coll. 2005;46(3):51-8. https://doi.org/10.2209/tdcpublication.46.51

21. Lago-Méndez L, Diniz-Freitas M, Senra-Rivera C, SeoanePesqueira G, Gándara-Rey JM, Garcia-Garcia A. Dental anxiety before removal of a third molar and association with general trait anxiety. J Oral Maxillofac Surg. 2006;64(9):1404-8. https://doi.org/10.1016/j.joms.2006.05.030

22. Fábián G, Müller O, Kovács S, Nguyen MT, Fábián TK, Csermely $\mathrm{P}$ et al. Attitude toward death: does it influence dental fear? Ann N Y Acad Sci. 2007;1113(1):339-49. https:// doi.org/10.1196/annals.1391.000

23. Locker D, Poulton R, Thomson WM. Psychological disorders and dental anxiety in a young adult population. Community Dent Oral Epidemiol. 2001;29(6):456-63. https://doi.org/10.1034/j.16000528.2001.290607.x

24. De Jongh A, Adair P, Meijerink-Anderson M. Clinical management of dental anxiety: what works for whom? Int Dent J. 2005;55(2):73-80. https://doi.org/10.1111/j.1875595X.2005.tb00037.x

25. Levin L, Eli I, Ashkenazi M. Dental anxiety among young Israeli male adults as related to treatment received during childhood. J Public Health Dent. 2006;66(2):147-51. https://doi. org/10.1111/j.1752-7325.2006.tb02571.x

26. Milgrom P, Newton JT, Boyle C, Heaton LJ, Donaldson N. The effects of dental anxiety and irregular attendance on referral for dental treatment under sedation within the National Health Service in London. Community Dent Oral Epidemiol. 2010;38(5):453-9. https://doi.org/10.1111/j.16000528.2010.00552.x

27. Heaton LJ, Carlson CR, Smith TA, Baer RA, de Leeuw R. Predicting anxiety during dental treatment using patients'self-reports: less is more. J Am Dent Assoc. 2007;138(2):188-95. https://doi.org/10.14219/jada. archive.2007.0135

28. Humphris G, King K. The prevalence of dental anxiety across previous distressing experiences. J Anxiety Disord. 2011;25(2):232-6. https://doi.org/10.1016/j. janxdis.2010.09.007

29. Quteish Taani DS. Dental anxiety and regularity of dental attendance in younger adults. J Oral Rehabil. 2002;29(6):604-8. https://doi.org/10.1046/j.13652842.2002.00905.x

30. Locker D, Thomson WM, Poulton R. Psychological disorder, conditioning experiences, and the onset of dental anxiety in early adulthood. J Dent Res. 2001;80(6):1588-92. https://doi.org/10.1177/0022034501 0800062201

31. Armfield JM. Predicting dental avoidance among dentally fearful Australian adults. Eur J Oral Sci. 2013;121(3 Pt 2):240-6. https://doi.org/10.1111/eos.12036 